\title{
Assessment of The Diagnostic Value of TOX Versus CD3 Immunohistochemical Markers in Detection of Early Mycosis Fungoides Cases \\ Mai Ahmed Gobran ${ }^{1}$, Ahmed Embaby ${ }^{2}$, Abeer Hafeez ${ }^{1}$ \\ Departments of ${ }^{1}$ Pathology and ${ }^{2}$ Internal Medicine, Faculty of Medicine, Zagazig university, Egypt \\ *Corresponding Author: Mai Ahmed Gobran, Email: magibran@medicine.zu.edu.eg
}

\begin{abstract}
Background: Primary cutaneous lymphoma (PCL) with clonal proliferation of atypical CD4+ skin-homing T lymphocyte cells is called Mycosis Fungoides (MF). TOX staining is observed in subtypes of PCL, as MF \& Sézary Syndrome (SS) in comparison to controls. Early MF is difficult to diagnose, \& its distinction from inflammatory diseases may be impossible.

Objective: This study aimed to evaluate the expression of the TOX versus CD3 as a diagnostic marker for early MF. Patients and methods: retrospective-cross sectional study includes $30 \mathrm{MF}$ and 30 benign cutaneous inflammatory diseases (BCID) cases. All were evaluated using $\mathrm{H} \& \mathrm{E}$ and immunohistochemical staining for TOX \& CD3.

Results: There was statistically significant increase of TOX \& CD3 expression in MF than BCID \& ability of TOX to detect all true positive cases $(100.0 \%)$ compared to $(76.7 \%)$ for $\mathrm{CD} 3,(\mathrm{P}<0.001)$.

Conclusion: TOX had the highest sensitivity (100.0\%) \& accuracy (88.3\%). TOX is useful marker in the diagnosis of early MF \& differentiating it from BCID.

Keywords: TOX, CD3, Mycosis Fungoides, Benign cutaneous inflammatory diseases (BCIDs), Immunohistochemistry.

\section{INTRODUCTION}

Atypical T-lymphocytes were found in patients with Mycosis Fungoides (MF), which is a primary cutaneous lymphoma (PCL). MF had the lengthy clinical progression, beginning with splatters and progressing through plaques to malignancies. In primary phases, MF mostly affects the skin, and among advanced MF cases, malignant lymphocytes can extend to the peripheral blood, lymph nodes, and visceral organs. The MF survival rate varies greatly depending on the stage of the disease.

Diagnosing MF is mostly depending on the combined algorithm of clinical and histological norms $(\mathbf{1}, \mathbf{2})$. The MF-histology shows a strip-like intervene of mononuclear cells within the papillary dermis with superimposing epidermotropism (intraepidermal lymphocytes with a spongiosis' paucity). These lymphocytes may be collectively or singly acquired within the epidermis, which were often enclosed by an obvious halo (Pautrier microabscesses). The highpower test of mononuclear cells shows hyperchromatic $\&$ unequal nuclear features. The epidermis normally displays an outline of psoriasiform epidermal hyperplasia with hyperkeratosis \& focal parakeratosis. Hematoxylin \& eosin-stained sections persist the diagnostic gold typical, but the diagnosis in the initial phase may be a challenge since it primarily may resemble the other chronic inflammatory dermatoses (3).

Thymocytes assortment equated HMG-box (TOX), is unfettered gene in the MF-skin biopsies, which encodes an extreme-motion group family (HMG) domain DNA-binding nuclear protein. This gene governs the developing T-cells differentiation ${ }^{(4)}$. TOX is initially expressed in the thymus and down-

controlled before exiting the CD4+ T-cells from the thymus. The proteins of TOX-mRNA were inadequately expressed in peripheral lymphoid tissue (5).

The stanning of TOX is expressed in several CTCLs-subtypes, i.e., peripheral T-cell lymphoma, $\mathrm{MF}$, and sézary syndrome (SS) and others, which were not detailed (PTCL-NOS) ${ }^{(\boldsymbol{\sigma})}$. In CTCL, miR-223 targeted a gene called TOX ${ }^{(7)}$. Furthermore, studies have shown that the TOX gene is highly expressed in the initial MF lesion as compared to controls ${ }^{(8)}$. A number of malignancies, including breast, leukemia, and lungs have been shown to have aberrant expression of the TOX-gene ${ }^{(\mathbf{9})}$.

Meanwhile, MF is not easy to diagnose, particularly at the initial lesions, where the uniqueness between inflammatory diseases and MF is occasionally unattainable. It was found that the infiltrating lymphocytes in MF are mostly CD4, with a marginal of CD8 T-cells. The CD4 is also expressed by histiocytes, which could be abundant herein ${ }^{(\mathbf{1 0})}$. The high ratio of CD4:CD8 is noted in typical initial phase of MF, where this ratio was furtherly declined. Nevertheless, there are uncommon MF-variants with the declines of CD4:CD8 proportion. The CD3 is the key reliably positive indicator for T-cells. The declined expression of CD7 could be improper but not definite for MF-diagnosis ${ }^{(11)}$.

However, it should be noticed that the CD7-loss could happen in harmless processes and other kinds of cutaneous T-Cell lymphoma (CTCL) ${ }^{(12)}$. The diagnostic measures of MF contain CD3-expression with $<50 \%$ of T-cells, CD7-expression with $<10 \%$ of T-cells, and epidermal disagreement from CD3- or CD7-expression on the dermal T-cells ${ }^{(13)}$.
\end{abstract}




\section{PATIENTS AND METHODS}

In this investigation, punch biopsy samples were obtained from 60 different patients and used in a retrospective, cross-sectional manner. The selected patients were identified as $30 \mathrm{MF}$-cases at the initial patch and plaque stages, and 30 BCIDs-cases grouped from the Pathology Department \& Dermatology Department, Faculty of Medicine, Zagazig University Hospitals within the duration starting from April 2017 till April 2020. The chosen samples were acquired by punch biopsy.

\section{Ethical approval:}

All patients agreed to biopsy acquiring and the agreement was assessed and permitted by IRB (Institutional Research Board-Faculty of MedicineZagazig University approval \#6967). This work has been carried out in accordance with The Code of Ethics of the World Medical Association (Declaration of Helsinki) for studies involving humans.

The clinical data regarding sex and age were also acquired from the patients' records. Paraffin blocks of all patients were divided at thickness of 3-4 m, stained with regular Hematoxylin, stained with eosin to reestimate, and the diagnosis was confirmed. Sequential sections from the equivalent blocks were delivered for immunohistochemical staining for TOX \& CD3.

Inclusion criteria: 1) Patients with active lesion of early- MF stage, and 2) Patients with active lesion of benign inflammatory dermatosis.

Omission criteria: 1) Pregnant \& lactating women, 2) Patients with tumor MF-stage, and 3) Patients with other kinds of cutaneous B and/or T-cell lymphoma.

\section{Histopathologic examination:}

The 30 cases of MF-related lesions included 20 cases with MF-patch phase and 10 cases with MFplaque phase. The 30 BCIDs-cases included 15 with chronic dermatitis, 10 with psoriasis, and 5 with lichen planus.

\section{Immunohistochemical staining:}

Using (Dako-Cytomation, Glostrup, Denmark) an early streptavidin-biotin immunoperoxidase technique to do the immunohistochemical staining. The slices had a thickness of 3-4 $\mathrm{mm}$ and were shown on positively charged slides after they had been formalinfixed and paraffin-inserted, rehydrated in rated $\mathrm{EtOH}$ after deparaffinization in xylene. Twenty minutes of boiling in citrate buffer ( $\mathrm{pH} \mathrm{6.0)}$ and then washing in PBS ( $\mathrm{pH}$ 7.3) were used to prepare the cross sections. Subsequently, blocking of endogenic peroxidase activity with $6 \%$ of $\mathrm{H} 2 \mathrm{O} 2$ in $\mathrm{MeOH}$ was done. The glides were then hatched overnight with monoclonal antibodies: TOX: rabbit polyclonal antibody or dilution 1:50 (Clone TOX, lab vision corporations). The positive controls for TOX were strong nuclear positivity in the germinal center and interfollicular zone. Meanwhile, the negative control was acquired by primary antibody omission.

CD3: Mouse monoclonal antibody, dilution1:100; (Clone 4A4, lab vision corporation, Santa Cruz, California, USA) as a chromogen, secondary antibody incubation was performed (Dako-Cytomation) using a diaminobenzidine substrate (from Research Genetics in Huntsville, Alabama, USA). By BioGenex Laboratories, San Ramon, California, USA, we counterstained the slides with Mayer's Hematoxylin and rinsed them with $\mathrm{dH} 2 \mathrm{O}$ and PBS twice. In both the germinal center and interfollicular zone were positive controls for CD3 (cytoplasmic positivity). The negative control was acquired by detach the primary antibody.

\section{Evaluating the immunohistochemical staining results:}

TOX \& CD3 expression were evaluated by totaling the cells number with brown staining nuclear for TOX and membranous for CD3 corresponds to the total amount of lymphocytes that have been accumulated. For semi-quantitative purposes, the staining can be classified as negative (less than 10\%), weak positive (10-30\%), moderately positive (between $11 \%$ and $50 \%$ ) or strong (more than 50\%) of the total infiltrating lymphocytes. The slides were evaluated by 2 pathologists operating individually. All inconsistencies were argued, and consent attained.

\section{Statistical analysis}

SPSS was used to do the statistical analysis (Chicago, IL, USA). For quantitative variables, the results were calculated as mean \pm standard deviation. It was decided to use Fisher's exact test or chi-square for categorical variables. The significance level was set at $\mathrm{P} \leq 0.05$. When it comes to discrete/count dependent variables or permanent dependent variables that deviate from the regularity assumption, the MannWhitney u test was used to see if there is a difference between medians of two independent groups.

\section{RESULTS}

The studied cases' age fluctuated from 20 to 55 years and the mean was $38.8 \pm 11.6$ years for BCIDsgroup, while, it was $41.8 \pm 9.0$ years for MF-group. The studied cases majority were males (57\%) in MF, and $60 \%$ with benign cutaneous inflammatory disorders (BCID). Both TOX and CD3 immunohistochemical expression were significantly different. The MF patients were found to be higher than that of BCIDs. The TOX-intensity of staining was intense in $70.0 \%$ of MF, while with only $6.7 \%$ in the BCID-group. While CD3 intensity of staining was strong in $66.7 .0 \%$ of MF group and only $43.3 \%$ of BCID group with statistically significant $(\mathrm{P} \leq 0.05)$ results (Table I). Regarding the diagnostic functioning of both indicators, we noted that the capability of TOX is $100.0 \%$, to identify all actual positive patients. Additionally, the TOX had the utmost sensitivity and 
accuracy with values of 100 and $88.3 \%$, respectively

(Table 2).

Table (1): Comparing TOX \& CD3 immuno-histochemical expression in MF patients versus benign cutaneous inflammatory disorders (BCIDs)

\begin{tabular}{|c|c|c|c|c|c|c|c|}
\hline \multirow{2}{*}{\multicolumn{2}{|c|}{$\begin{array}{c}\text { Immunohistochemical } \\
\text { expression }\end{array}$}} & \multicolumn{2}{|c|}{ MF CASES } & \multicolumn{2}{|c|}{ BCID } & \multirow{2}{*}{$\chi^{2}$} & \multirow[b]{2}{*}{ p-value } \\
\hline & & No & $\%$ & No & $\%$ & & \\
\hline \multirow{4}{*}{$\begin{array}{c}\text { CD3 } \\
\text { immunosta } \\
\text {-ining }\end{array}$} & Negative & 7 & 23.3 & 1 & 3.3 & \multirow{4}{*}{14.8} & \multirow{4}{*}{$0.002 ”$} \\
\hline & Weak positive & 2 & 6.7 & 10 & 33.3 & & \\
\hline & $\begin{array}{l}\text { Moderate } \\
\text { positive }\end{array}$ & 1 & 3.3 & 6 & 20.0 & & \\
\hline & Strong Positive & $\overline{20}$ & 66.7 & 13 & 43.3 & & \\
\hline \multirow{4}{*}{$\begin{array}{l}\text { TOX- } \\
\text { staining }\end{array}$} & Negative & 0 & 0.0 & 23 & 76 & \multirow{4}{*}{$X 2=42.6$} & \multirow{4}{*}{$<0.001 *$} \\
\hline & Weak positive & 0 & 0.0 & 3 & 10 & & \\
\hline & $\begin{array}{l}\text { Moderate } \\
\text { positive }\end{array}$ & 9 & 30 & 2 & 6.7 & & \\
\hline & Strong positive & 21 & 70 & 2 & 6.7 & & \\
\hline
\end{tabular}

Table (2): Diagnostic performance of TOX \& CD3 in detecting early MF

\begin{tabular}{|l|c|c|c|c|c|}
\hline Variable & Sensitivity & Specificity & PPV & NPV & Accuracy \\
\hline TOX & $100.0 \%$ & $76.7 \%$ & $81.1 \%$ & $100.0 \%$ & $88.3 \%$ \\
\hline CD3 & $76.7 \%$ & $3.3 \%$ & $44.2 \%$ & $12.5 \%$ & $40.0 \%$ \\
\hline
\end{tabular}

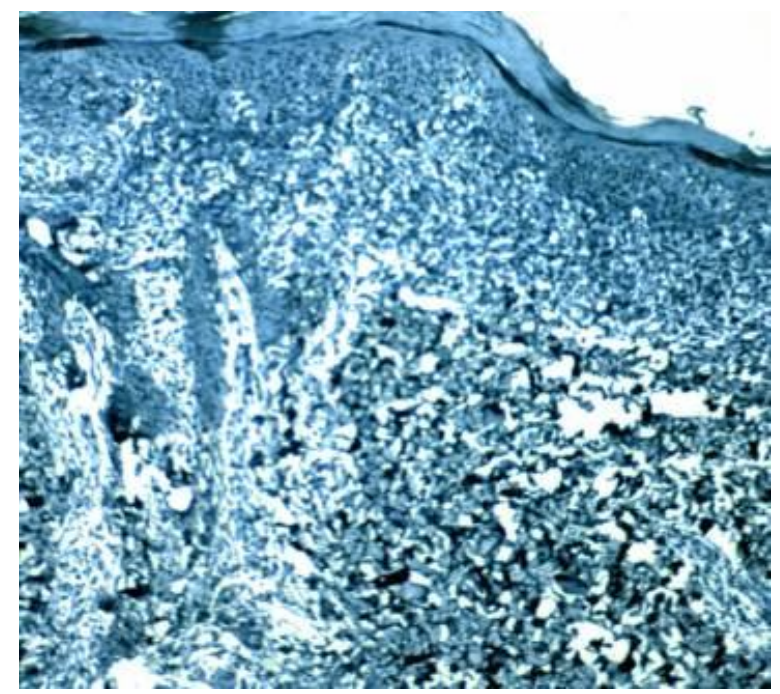

Figure (1): A case of chronic dermatitis showing negative TOX immunoreactivity (Immunoperoxidase stain, X 100)

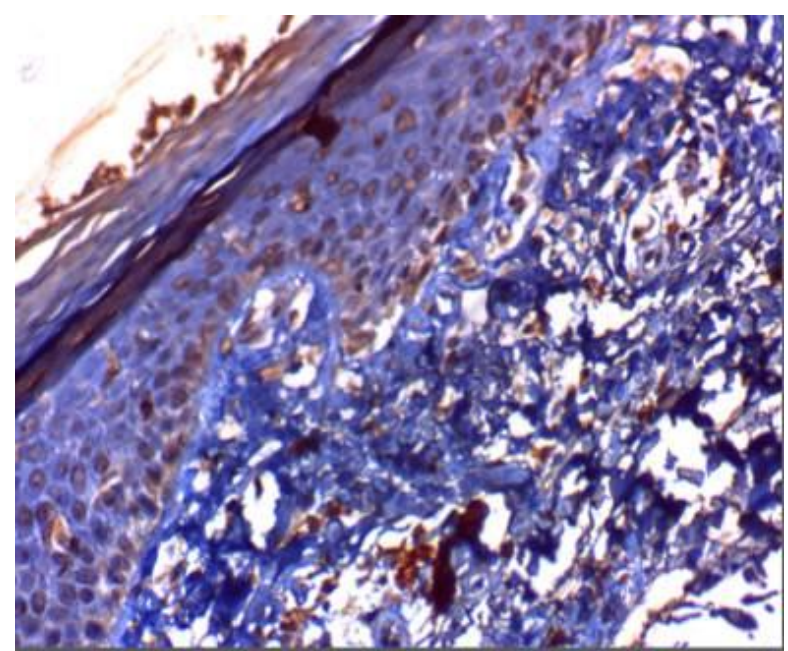

Figure (2): A. case of MF showing strong positivity of TOX immunoreactivity (Immunoperoxidase stain, X 400) 


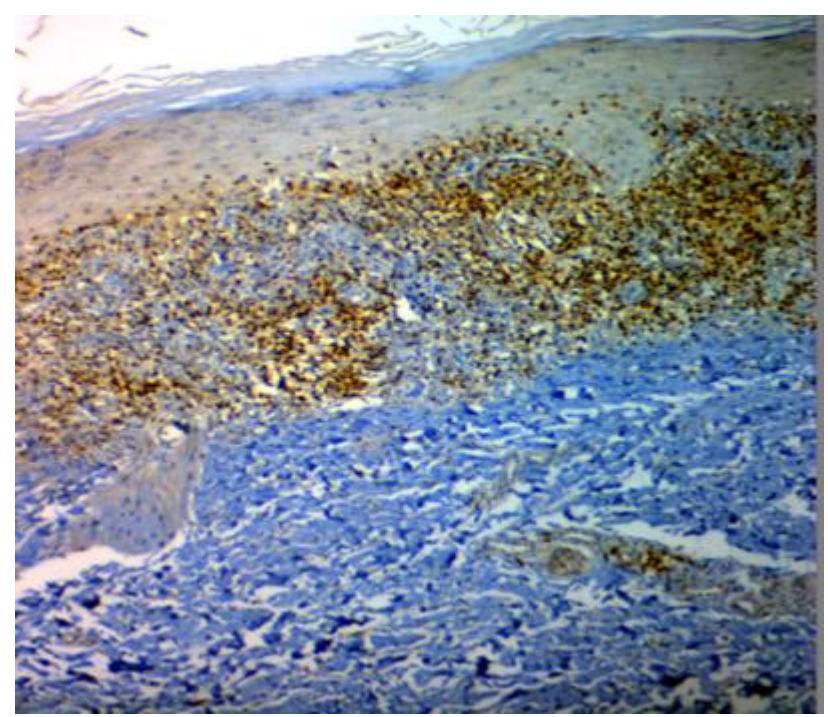

Figure (3): A case of lichen planus showing strong positivity of CD3 immunoreactivity (Immunoperoxidase stain, $\mathrm{X}$ 100)

\section{DISCUSSION}

The 30 cases of MF-related lesions comprised of 20 MF-cases at the patch phase and 10 cases of plaque MF-phase, while the 30 BCIDs-cases included 15 with chronic dermatitis, 10 with psoriasis, and 5 with lichen planus cases.

Herein, we created a proportional analysis of the initial stage of MF versus BCIDs with regards of TOX and $\mathrm{CD} \quad 3 \quad \mathrm{~T}$-cell subsets immuno-histochemical expression. The $30 \mathrm{MF}$ and $30 \mathrm{BCIDs}$ patients were included herein. Our findings disclosed that both MF and BCIDs cases were more communal in males with rates of $57 \%$ and $60 \%$ than in females with rates of $43 \%$ and $40 \%$, respectively with a statistical insignificant result $(\mathrm{P}=0.7)$.

MF lesions versus BCID samples showed substantial expression of the TOX thymocyte selectionrelated high mobility group box factor. High mobility group (HMG) family nuclear protein encoded by this gene functions as a transcription factor. Thymic tissue expresses it at high levels but for a short time. During thymocyte differentiation, particularly during CD4 CD8 precursor to $\mathrm{CD} 4+\mathrm{T}$-cell transition, the $\mathrm{TOX}$ is a crucial director of early T-cell progression. The typical mature of CD4-cells hardly have significant expression of TOX-protein. The abnormal TOX expression in MF is approved in initial and late stages of MF without significant expression in the chronic dermatitis. The TOX could serve as a diagnostic and prognostic sign for people with $\mathrm{MF}^{(14)}$.

In our study, we found that there was a significant higher expression of TOX in MF-group than in BCIDsgroup. The staining intensity was deep in $70.0 \%$ of MFgroup, while only in $6.7 \%$ of BCIDs-group with a statistically significant result $(p=0.001)$. According to these findings, TOX was found to be overexpressed in MF-lesions by Huang et al. ${ }^{(14)}$ and Yu et al. ${ }^{(4)}$. The TOX is a diagnostic marker for MF, and it may serve as a pathogenic component in the course of the disease. In 32 of $35(91 \%)$ biopsies, the neoplastic T-cells disclosed a clear nuclear, whereas only 6 of $30(20 \%)$ of BCID specimens displayed a positive staining. A significant difference between $\mathrm{MF}$ and BCID $(\mathrm{P}<0.0001$, Chi-square test) was also found. Our results disclosed a significant difference in TOX-expression between MF and BCID, where the TOX in MF were also higher than that in BCID. Refat et al. ${ }^{(15)}$ reported that MF-cases showed higher significant TOXexpression than compared to the inflammatory dermatoses, with 100 percent of sensitivity and 62 percent of specificity. McGirt et al. ${ }^{(16)}$ also noted that MF had been peaked the TOX-expression in $73.6 \%$ of MF cases compared to $31.6 \%$ in BCIDs.

The neoplastic lymphocytes of MF always display a cluster of differentiation $\mathrm{CD} 3+, \mathrm{CD} 4+, \mathrm{CD} 5+, \mathrm{CD} 8-$, CD45Ro+, and T-cell intracellular antigen (TIA)-1-. In tumoral lesions, there may be missing of pan-T-cell antigens (CD2, CD3, and CD5) ${ }^{(17)}$.

Herein, a statistically higher CD3 immunohistochemical expression in MF group than in BCIDs group was noted. The staining intensity was strong in $66.7 .0 \%$ of MF, and only $43.3 \%$ of BCIF group with a statistically significant result $(\mathrm{P} \leq 0.05)$. Cerroni et al. ${ }^{(18)}$ and Kelati et al. ${ }^{(19)}$ also indorsed positive CD3-expression in MF-cases. It was identified the following diagnostic characteristics to differentiate MF from benign dermatosis, including superficial lymphoid infiltrate, clear cytoplasm (haloed cells), enlarged hyperchromatic cribriform nuclei, and immunohistochemistry, which was positive for CD3. However, Burg et al. ${ }^{(20)}$ and Pimpinelli et al. ${ }^{(21)}$ noted that the loss of pan-T-cell indicator (CD3) in epidermal, but not dermal lymphocyte, implying MF-diagnosis. Although, the loss of these markers noted also in inflammatory conditions, but it is less massive than in MF. The causes of conflict results may be owing to the stages of MF.

Regarding the diagnostic performing of both markers, we noted that there was a statistically significant difference between MF and BCIDs groups in TOX and in CD3 immunohistochemical expression. The capability of TOX to identify all true positive cases 
was $100.0 \%$, TOX-specificity was $76.7 \%$, positive predictive value of TOX was $81.1 \%$, negative predictive value of TOX was $100 \%$, TOX-accuracy was $88.3 \%$, TOX- sensitivity was $100.0 \%$, and TOXaccuracy was $88.3 \%$.

The capability of CD3 to identify the true positive cases was $76.7 \%$, CD3-sensitivity was $76.7 \%$, CD3specificity was $3.3 \%$, CD3 positive and negative predictive values were 44.2 and $12.5 \%$ respectively and CD3-accuracy was $40 \%$. Refat et al. ${ }^{(15)}$ and Morimura et al. ${ }^{(6)}$ agree with our results, where TOX had $62 \%$ and $100 \%$ sensitivity and specificity for MF respectively. In addition, McGirt et al. ${ }^{(16)}$ also matches that TOX positive predictive value (PPV) for any TOXexpression (Grade 1-3) was about $86.7 \%$, where the negative predictive value was $48.1 \%$. A strong TOXexpression (Grade 2-3) was also identified in 33 of 53 MF-cases (62.3\%), which had a PPV of $97.1 \%$ and a NPV of $47.4 \%$. Inversely, a positive TOX-expression is noticed in 6 of $19(31.6 \%)$ of BCID/NS samples, with a strong positive TOX-expression was detected in only 1 of $19(5.3 \%)$ of BCID-samples. However, inferior specificity (75\%) and superior sensitivity $(90.3 \%)$ was noted by Hung et al. ${ }^{(14)}$, as both worked on larger number of cases with different methodology at genetic level and lower cut-off value.

Pimpinelli et al. ${ }^{(21)}$ also reported that the loss of CD3 in T-cells is only $10 \%$ sensitive despite its $100 \%$ specificity, though their shortage is involved in the ISCL criteria to describe the early MF.

\section{CONCLUSION}

The TOX could be deemed as a valuable marker in MF-diagnosis with sensitivity of $100 \%$, specificity of $76.7 \%$ and accuracy of $88.3 \%$. It is more definite and valued in distinguishing MF from BCID than CD3.

\section{Limitations:}

Further studies on TOX specificity as it could be expressed in other types of cutaneous lymphomas not only in mycosis fungoides. CD3 is lost in some mycosis fungoides subtypes, as we included only classic MF patch and plaque stages. So, the use of other CD markers such as CD5, CD7, CD4, CD8 in future studies can be helpful in preliminary diagnosis of MF.

\section{Financial support and sponsorship: Nil. Conflict of interest: Nil.}

\section{REFERENCES}

1. Willemze R, Jaffe E, Burg G et al. (2005): WHOEORTC classification for cutaneous lymphomas. Blood, 105: 3768-3785.

2. Olsen E, Vonderheid E, Pimpinelli $N$ et al. (2015): Revisions to the staging \& classification of MF \& Sezary syndrome: European Organization of Research \& Treatment of Cancer (EORTC). Blood, 110: 1713-1722.

3. Connie A, Iriana P, Belongie G et al. (2007): The Diagnosis, Staging, \& Treatment Options for mycosis fungoides. Cancer Control, 14 (2): 102-111.
4. Yu X, Luo Y, Liu J et al. (2015): TOX acts an Oncological Role in MF. PLoS One, 10 (3): 1174-79.

5. Wilkinson B, Chen J, Han $P$ et al. (2002): TOX: an HMG box protein implicated in the regulation of thymocyte selection. Nat Immunol., 3: 272-280.

6. Morimura S, Sugaya M, Suga H et al. (2014): TOX expression in different subtypes of cutaneous lymphoma. Arch Dermatol Res., 306: 843-9.

7. McGirt L, Adams C, Baerenwald D et al. (2014): miR-223 regulates cell growth \& targets protooncogenes in MF/cutaneous T-cell lymphoma. J Invest Dermatol., 134: 1101-1107.

8. Zhang Y, Wang Y, Yu R et al. (2012): Molecular markers of early-stage mycosis fungoides. $\mathbf{J}$ Invest Dermatol., 132: 1698-1706.

9. Gonzalez-Aguilar A, Idbaih A, Boisselier B et al. (2012): Recurrent mutations of MYD88 \& TBL1XR1 in primary central nervous system lymphomas. Clin Cancer Res., 18: 5203-5211.

10. Ortonne $\mathrm{N}$, Buyukbaban $\mathrm{N}$, Delfau-Larue $\mathrm{M}$ et al. (2003): Value of the CD8-CD3 ratio for the diagnosis of MF. Mod Pathol., 16: 857-862.

11. Campbell S, Peters S, Zirwas $M$ et al. (2010): Immunophenotypic Diagnosis of Primary Cutaneous Lymphomas: A Review for the Practicing Dermatologist. The Journal of Clinical \& Aesthetic Dermatology, 3 (10): 21-25.

12. McPherson R (2001): Overview of the immune system \& immunologic disorders. In: McPherson RA, Pincus MR. Henry's Clinical Diagnosis \& Management by Laboratory Methods. 20th ed. Philadelphia, Pa: WB Saunders Co., Pp: 817-820.

13. Olsen E, Vonderheid E, Pimpinelli D et al. (2015): Revisions to the staging \& classification of MF \& Sezary syndrome: European Organization of Research \& Treatment of Cancer (EORTC). Blood, 110: 1713-1722.

14. Hung T, Yeung T, Cherng-Lih $P$ et al. (2017): Folliculotropic MF: A case after one-year follow-up. Dermatologica Sinica., 35: 206-209.

15. Refat S, EL-Shawaf I, Abd EL- Hameed A et al. (2015): The Value of TOX as a Diagnostic marker for mycosis fungoide. ZUMJ., 21: 1-5.

16. McGirt L, Degesys C, Johnson V et al. (2016): TOX expression \& role in CTCL. J Eur Acad Dermatol Venereol., 30 (9): 1497-1502.

17. Scarisbrick J, Hodak E, Bagot M et al. (2018): Blood classification \& blood response criteria in MF \& Sézary syndrome using flow cytometry: recommendations from the EORTC cutaneous lymphoma task force. Eur J Cancer, 93:47-56.

18. Cerroni L (2018): MF clinical \& histopathologic features, differential diagnosis, \& treatment. Seminars in Cutaneous Medicine \& Surgery, 37: 1085-1089.

19. Kelati A, Gallouj S, Tahiri L et al. (2017): Defining the mimics \& clinico-histological diagnosis criteria for MF to minimize misdiagnosis. International Journal of Womans Dermatology, 3: 100-106.

20. Burg G, Kempf W, Cozzio A et al. (2005): WHO/EORTC classification of cutaneous lymphomas 2005: Histological \& molecular aspects. J Cutan Pathol., 32: 647-674.

21. Pimpinelli N, Olsen E, Santucci M et al. (2005): Defining early mycosis fungoides. J Am Acad Dermatol., 53: 1053-63. 